\title{
Performance Analysis of a Delay-Tolerable Cognitive Radio Network
}

\author{
Shensheng TANG \\ Missouri Western State University \\ St. Joseph, MO 64506, USA \\ e-mail: stang@missouriwestern.edu
}

\author{
Chenghua TANG \\ Guilin University of Electronic Technology \\ Guilin, Guangxi Province, China \\ e-mail: tch@guet.edu.cn
}

\begin{abstract}
In this paper, we analyze the performance of a delay-tolerable cognitive radio network at the call level, where secondary users detect and opportunistically access the channel resources when no primary users present. An ongoing secondary user using a channel may switch to another channel with some routing probability (due to the presence of a primary call) or leaves the channel (due to service completion) with some other probability. If the target channel is being used at that time, the secondary call will be placed in the queue of the channel. The queued secondary calls will reconnect back to the system when the channel becomes available. We develop a queueing network model for the system and solve the equilibrium system states by the generating function technique. Numerical results are presented to show the impact of system parameters on the performance metrics.
\end{abstract}

Keywords-cognitive radio network; primary call; secondary call; opportunistic spectrum access

\section{INTRODUCTION}

In the past decade, intensive study on cognitive radio (CR) networks has been thoroughly aroused in academia, industry, and government agencies as to the high efficient spectrum improvement. The users in CR networks are equipped with CRs are referred to as secondary users, who are unlicensed users and opportunistically share the spectrum resources with the licensed users (i.e., primary users) in the same area. By allowing secondary users to reclaim idle channels, much higher spectrum efficiency can be achieved [1].

In CR networks, the spectrum availability for the secondary users depends on the spectrum occupancy of the primary users. A distinct feature of such networks is that the secondary users have the capability to sense channel usage and switch among different channels, without causing harmful interference on primary users. Therefore, each initiating secondary user is required to decide whether or not the channel is occupied by a primary user. On the other hand, for an ongoing secondary user, it still needs to sense the spectrum periodically, to achieve the channel-status information for its future use. The ongoing secondary user may leave its current channel either due to service completion or due to the presence of a primary call on the channel. Recent research on CR networks has been done using signal detection and estimation techniques [2, 3], probability and queuing theoretic techniques [4, 5], game theoretic methods [6, 7], data fusion methods [8], and other methods [9] as tools for investigating spectrum detection, spectrum selection and spectrum sharing aspects.

In [2], detection performance of an energy detector based cooperative spectrum sensing was investigated in a cognitive radio network over Rayleigh and Raleigh-lognormal fading channels. In [3], the sensing period was optimized to make full use of opportunities in the licensed bands. In [4], an analysis of reconnection opportunity was presented for the queued secondary calls in the buffer in a CR network under unreliable sensing. In [5], a spectrum decision model based on an M/G/1 model was proposed to evaluate the load-balancing of secondary users and effects of multiple interruptions from the primary user during each link connection. In [6], a tutorial survey was presented to introduce the basic concepts of game theory and explain in detail how these concepts can be leveraged in designing spectrum sharing protocols. In [7], a new approach based on Bayesian network and game theory was proposed to analyze the influence of incomplete data and uncertain information in CR networks. In [8], a centralized soft-combining data fusion algorithm based on the joint probability distribution of fourth order cumulants was presented for cooperative modulation classification in CR networks. In [9], two power adjustment methods (PID control and model predictive control) were proposed based on control theory to decide transmitter transmission power for CR networks.

In this paper, we analyze the performance of a CR network in which the secondary traffic is delay tolerable. The CR network is modeled using a queueing network, where secondary calls are never blocked due to delay toleration and enter a queue associated with every channel in case the channel is busy. A secondary call waits in the queue until the channel becomes available. Thus, the queueing network model is appropriate for delay-tolerant data traffic. The main contribution of this paper is the formulation of a CR data network in terms of a queueing network model and the solution of the system state probabilities through the generating function technique. The analytical results derived from the queueing network model can be used to dimension secondary traffic flows subject to constraints imposed by the primary traffic.

The remainder of the paper is organized as follows. Section 2 describes a queueing network model of the CR data network. Section 3 develops the analysis for a given channel by considering the channel occupancy characteristics of primary and secondary calls. Section 4 presents numerical results. Finally, the paper is concluded in Section 5. 


\section{SYSTEM MODEL}

In the CR network, secondary users are able to sense channel usage and switch between different channels using appropriate communication mechanisms and time-sampled signal-strength measurements, while causing no harmful interference to the primary users. The spectrum band is divided into $N$ frequency channels serving primary users and opportunistically being shared with secondary users via reliable sensing. Primary users operate as if there are no secondary users in the service area. Primary calls arriving to different channels form independent Poisson processes. An incoming primary call occupies a channel if it is free; otherwise, the call is blocked.

A secondary user has to sense the channel availability before accessing a channel. If it detects that a channel is being used, it can choose to either enter a queue associated with the channel for waiting or continue to sense another channel.

After a secondary user accesses an idle channel, say channel $i$, he/she has to continue sensing the channel in case a primary user attempts to use the channel. This secondary call may switch to another channel, say $j$, with probability $r_{i j}$ or complete without interruption and leaves the system with probability $r_{i 0}$, where $r_{i 0}=1-\sum_{j=1}^{N} r_{i j}, j \neq i$. If channel $j$ is being used at that time, the secondary call joins the queue associated with channel $j$. The queued secondary calls access the channel in FCFS (first-come first-served) manner as the channel becomes available.

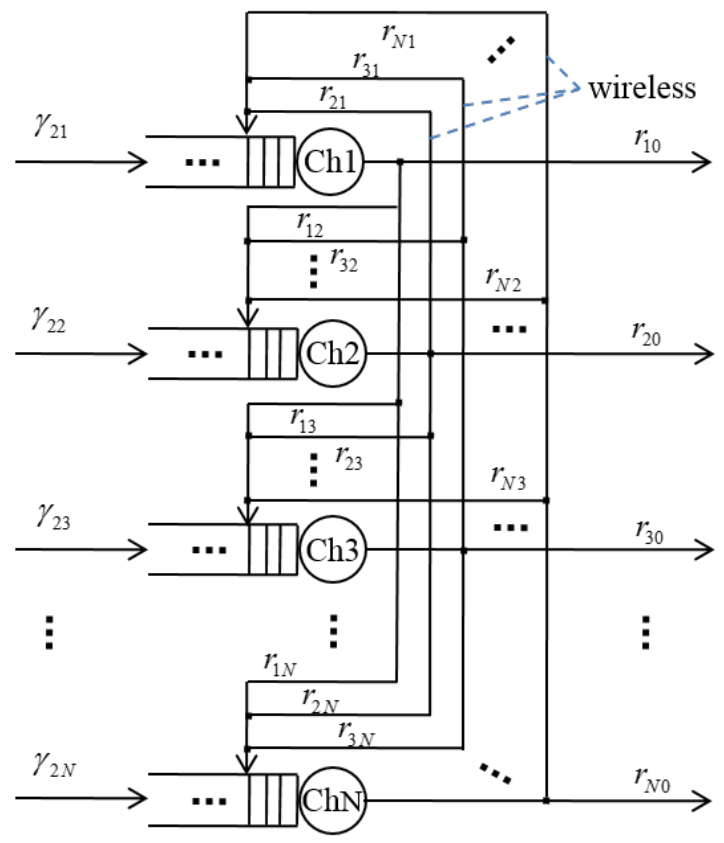

Figure 1. Queueing network model of the CR network

Fig. 1 shows the proposed queueing network model of the CR network, where each channel is effectively a single server queueing system. The external arriving secondary calls to channel $i$ form a Poisson process with rate $\gamma_{i}, 1 \leq i \leq N$.
The service time of secondary calls at channel $i$ is assumed to be exponentially distributed with parameter $\mu_{2 i}$. The dynamics of the primary calls are not shown explicitly in the figure, however, the constraints imposed by the primary calls on the secondary calls are embedded in the model. For example, routing probabilities depend on the activity of the primary users. From Fig. 1, the total secondary call arrival rate to channel $i, \lambda_{2 i}$, can be obtained from the external arrival rate $\gamma_{i}$ and the internal arrival rates from other channels. When the system is in steady-state, the output rate of channel $\mathrm{i}$ is equal to its arrival rate. Hence, we have the following traffic equation [10]: $\lambda_{2 i}=\gamma_{i}+\sum_{j=1}^{N} \lambda_{2 j} r_{j i}, j \neq i, 1 \leq i \leq N$, where $r_{j i}$ is the routing probability from channel $j$ to $i$. This traffic equation can be expressed in vector form:

$$
\Lambda_{S}=\Gamma_{S}+P^{\prime} \Lambda_{S},
$$

where $\quad \Lambda_{S}=\left(\lambda_{21}, \lambda_{22}, \cdots, \lambda_{2 N}\right)^{\prime}$, $\Gamma_{S}=\left(\gamma_{1}, \gamma_{2}, \cdots, \gamma_{N}\right)^{\prime}$, and the routing matrix is

$$
P=\left[\begin{array}{cccc}
0 & r_{12} & \cdots & r_{1 N} \\
r_{21} & 0 & \cdots & r_{2 N} \\
\cdots & \cdots & \cdots & \cdots \\
r_{N 1} & r_{N 2} & \cdots & r_{N N}
\end{array}\right] .
$$

Since the matrix $\left(I-P^{\prime}\right)$ is invertible, (1) has a unique solution as follows:

$$
\Lambda_{S}=\left(I-P^{\prime}\right)^{-1} \Gamma_{S}
$$

\section{PERFORMANCE ANALYSIS}

In this section, we analyze the performance of the queue associated with an arbitrary channel $i$ under reliable sensing. A single server queue is maintained for secondary calls waiting to be served by channel $i$. We first analyze the routing probabilities and then the system performance.

Let the arrival process of primary calls to channel $i$ be Poisson with rate $\lambda_{1 i}$ and the service rate $\mu_{1 i}$. Then the interarrival time $X_{i}$ of primary calls to channel $i$ is exponentially distributed with mean $1 / \lambda_{1 i}$. Let random variable $R_{i}$ be the time duration between the instant that a primary call completes its service and the instant that the next primary call arrives (i.e., $R_{i}$ is the residual time of $X_{i}$ ). By memoryless property, the probability density function (pdf) of $R_{i}$ can be obtained as 


$$
f_{R_{i}}(t)=\lambda_{1 i} \exp \left(-\lambda_{1 i} t\right)
$$

Let $Y_{i}$ be the requested service time of secondary calls to channel $i$, then, according to the previous assumption, $Y_{i}$ is exponentially distributed with mean $1 / \mu_{2 i}$. When $R_{i}>Y_{i}$, the secondary call can complete its service and leave the system before a primary call arrives. Thus, the probability that a secondary call leaves the system, $r_{i 0}$, is

$r_{i 0}=P\left(R_{i}>Y_{i}\right)=\int_{0}^{\infty} \int_{t}^{\infty} f_{Y_{i}}(t) f_{R_{i}}(\tau) d \tau d t=\mu_{2 i} /\left(\lambda_{1 i}+\mu_{2 i}\right)$

When $R_{i}<Y_{i}$, the secondary call will have to move out from its current channel before completing its service. Similarly, we have $P\left(R_{i}<Y_{i}\right)=\lambda_{1 i} /\left(\lambda_{1 i}+\mu_{2 i}\right)$, the probability that a secondary call moves out from its current channel due to the presence of a primary call. Assume the secondary call enters some channel $j$ with probability $\beta_{i j}$, given that it moves out from its current channel $i$, where $\sum_{j=1}^{N} \beta_{i j}=1, j \neq i$. Then, the routing probabilities $r_{i j}$ is

$$
r_{i j}=\lambda_{1 i} \beta_{i j} /\left(\lambda_{1 i}+\mu_{2 i}\right)
$$

where $\beta_{i j}$ can be determined based on an appropriate channel selection scheme or the channel status information maintained in cognitive radios, which is updated periodically via spectrum sensing. For simplicity, we choose the equal-probability channel assignment strategy, i.e., $\beta_{i j}=1 /(N-1)$, which can be substituted into (6) to evaluate $\Lambda_{S}$ in (3).

Let $\{K(t), J(t)\}$ represent the state of the queue associated with channel $i$ at time $t$, where $K(t)$ is set

to 1 if the system is in the primary mode serving a primary call, 0 if the system is in the secondary mode serving a secondary call, and $J(t)$ denotes the number of secondary calls in the system including the one in service (if any). The process $\{K(t), J(t)\}$ is a two-dimensional Markov process with state space $\{(k, j) \mid k=0,1 ; j=0,1,2, \cdots\}$ and state transition diagram is shown in Fig. 2.

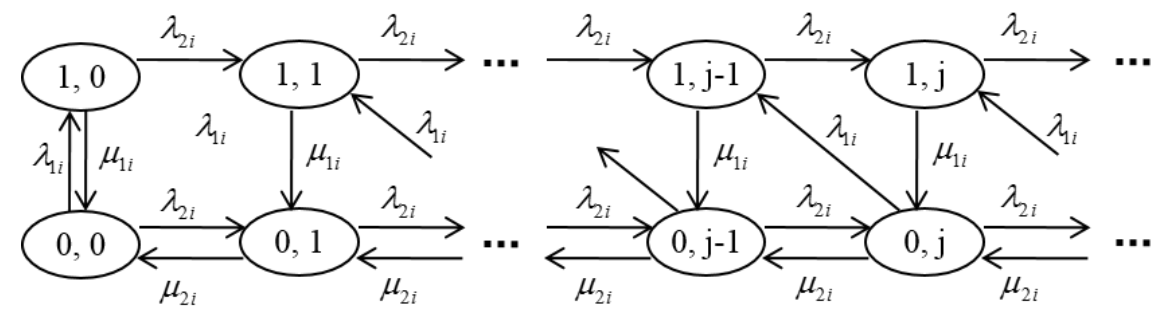

Figure 2. State transition diagram of the channel i system

Let $\pi_{i}(k, j)$ denote the steady-state probability that the channel $i$ system is in state $(k, j)$. Note that state $(0,0)$ denotes an empty system. The global balance equations of the system are as follows:

$$
\begin{gathered}
\pi_{i}(1,0)\left(\lambda_{2 i}+\mu_{1 i}\right)=\pi_{i}(0,0) \lambda_{1 i}+\pi_{i}(0,1) \lambda_{1 i}, \\
\pi_{i}(0,0)\left(\lambda_{1 i}+\lambda_{2 i}\right)=\pi_{i}(0,1) \mu_{2 i}+\pi_{i}(1,0) \mu_{1 i}, \\
\pi_{i}(1, j)\left(\lambda_{2 i}+\mu_{1 i}\right)=\pi_{i}(0, j+1) \lambda_{1 i}+\pi_{i}(0, j-1) \lambda_{2 i}, j \geq 1, \\
\pi_{i}(0, j)\left(\lambda_{1 i}+\lambda_{2 i}+\mu_{2 i}\right)=\pi_{i}(0, j+1) \mu_{2 i}+\pi_{i}(0, j-1) \lambda_{2 i}+\pi_{i}(1, j) \mu_{1 i}, j \geq 1,
\end{gathered}
$$

We use generating function technique [11] to solve the above system equations. Define the partial generating functions of the system as

$$
G_{k, i}(z)=\sum_{j=0}^{\infty} \pi_{i}(k, j) z^{j}, \quad|z| \leq 1, k=0,1
$$


Applying $G_{k, i}(\mathrm{z})$ to the above system equations, we obtain the following equations:

$$
\begin{gathered}
G_{0, i}(z) \lambda_{1 i}+G_{1, i}(z)\left[\lambda_{2 i} z^{2}-\left(\lambda_{2 i}+\mu_{1 i}\right) z\right]=\pi_{i}(0,0) \lambda_{1 i}(1-z), \\
G_{0, i}(z)\left[\lambda_{2 i} z^{2}-\left(\lambda_{1 i}+\lambda_{2 i}+\mu_{2 i}\right) z+\mu_{2 i}\right]+G_{1, i}(z) \mu_{1 i} z=\pi_{i}(0,0) \mu_{2 i}(1-z) .
\end{gathered}
$$

Solving (12) and (13), we have

$$
\begin{gathered}
G_{0, i}(z)=\pi_{i}(0,0)\left(\lambda_{1 i} \mu_{1 i}+\lambda_{2 i} \mu_{2 i}+\mu_{1 i} \mu_{2 i}-\lambda_{2 i} \mu_{2 i} z\right) / D(z), \\
G_{1, i}(z)=\pi_{i}(0,0) \lambda_{1 i}\left(\lambda_{1 i}+\lambda_{2 i}+\mu_{2 i}-\lambda_{2 i} z\right) / D(z)
\end{gathered}
$$

where $D(z)=\lambda_{2 i}{ }^{2} z^{2}-\lambda_{2 i}\left(\lambda_{1 i}+\lambda_{2 i}+\mu_{1 i}+\mu_{2 i}\right) z+\lambda_{1 i} \mu_{1 i}+\lambda_{2 i} \mu_{2 i}+\mu_{1 i} \mu_{2 i}$. Using the normalization condition $G_{0, i}(1)+G_{1, i}(1)=1$, we have

$$
\pi_{i}(0,0)=\frac{\lambda_{1 i}\left(\mu_{1 i}-\lambda_{2 i}\right)+\mu_{1 i}\left(\mu_{2 i}-\lambda_{2 i}\right)}{\left(\lambda_{1 i}+\mu_{1 i}\right)\left(\lambda_{1 i}+\mu_{2 i}\right)} \text {, and } \pi_{i}(1,0)=\frac{\lambda_{1 i}\left(\lambda_{1 i}+\lambda_{2 i}+\mu_{2 i}\right)}{\lambda_{1 i} \mu_{1 i}+\lambda_{2 i} \mu_{2 i}+\mu_{1 i} \mu_{2 i}} \pi_{i}(0,0) \text {. }
$$

In practice, $0<\pi_{i}(0,0)<1$, thus the following condition is required for system stability:

$$
\frac{\lambda_{2 i}\left(\lambda_{1 i}+\mu_{1 i}\right)}{\mu_{1 i}\left(\lambda_{1 i}+\mu_{2 i}\right)}<1
$$

The partial generating functions $G_{k, i}(z), k=0,1$, are then determined by substituting (16) into (14) and (15). Based on which, we can derive some important performance metrics.

- Mean Number of Secondary Calls

The mean number of secondary calls in the channel $i$ system, denoted by $L_{i}$, can be derived as

$$
L_{i}=\sum_{k=0}^{1} \sum_{j=0}^{\infty} G_{k, i}^{\prime}(1)
$$

\section{- System Utilization and Contribution Factor of Secondary Calls}

The channel $i$ system utilization $\rho_{i}$ is the probability that the server is busy and given by

$$
\rho_{i}=1-\pi_{i}(0,0)
$$

of which the contribution percentage from the secondary calls, defined as the metric of contribution factor of secondary calls $\eta_{i}$, can be calculated as

$$
\eta_{i}=\frac{\rho_{i}-\pi_{i}(1,0)}{\rho_{i}}=1-\frac{\pi_{i}(1,0)}{\rho_{i}}
$$

\section{NUMERICAL RESULTS}

We present numerical results to validate the analysis by assuming a system with $N=12$ channels. The primary call arrival rate vector is $\boldsymbol{\Lambda}_{P}=\lambda_{P} \mathbf{v}_{P}$, and its associated service rate vector is $\mathbf{U}_{P}=\mu_{P} \mathbf{v}_{P}$; the external arrival rate vector of secondary calls is $\boldsymbol{\Gamma}_{S}=\gamma_{S} \mathbf{v}_{S}$, and the secondary call service rate vector is $\mathbf{U}_{S}=\mu_{S} \mathbf{v}_{S}$. The constant vectors $\mathbf{v}_{P}$ and $\mathbf{v}_{S}$ are randomly selected as follows:

$\mathbf{v}_{P}=[1.1,1.2,1.3,1.5,1.7,1.4,1.5,1.6,1.8,1.9,1.5,1.6]^{\prime} ;$ $\mathbf{v}_{S}=[1.7,1.9,1.9,1.8,2.1,1.9,1.8,2.2,2.3,2.4,1.9,2.0]^{\prime}$.

In the numerical results, the parameters $\lambda_{P}, \gamma_{S}, \mu_{P}$, and $\mu_{S}$ are either varied or set to default fixed values as follows: $\lambda_{P}=2$ or $3, \gamma_{S}=1, \mu_{P}=6$, and $\mu_{S}=12$ or 15 . Without loss of generality, we only show the numerical results for channel 1 system (i.e., $i=1$ ).

Fig. 3 shows the variation in the probability $r_{i 0}$ of secondary calls leaving channel 1 system when $\lambda_{P}$ and $\mu_{S}$ are both varied. We observe $r_{i 0}$ increases as $\lambda_{P}$ decreases or 
$\mu_{S}$ increases. When $\lambda_{P}$ is decreased, the channel will be occupied by primary calls less frequently and thus ongoing secondary calls will have more opportunities to complete their service and leave the system. Similarly, an increase in $\mu_{S}$ leads to more opportunities for secondary calls to complete their service before the next arrival of a primary call.

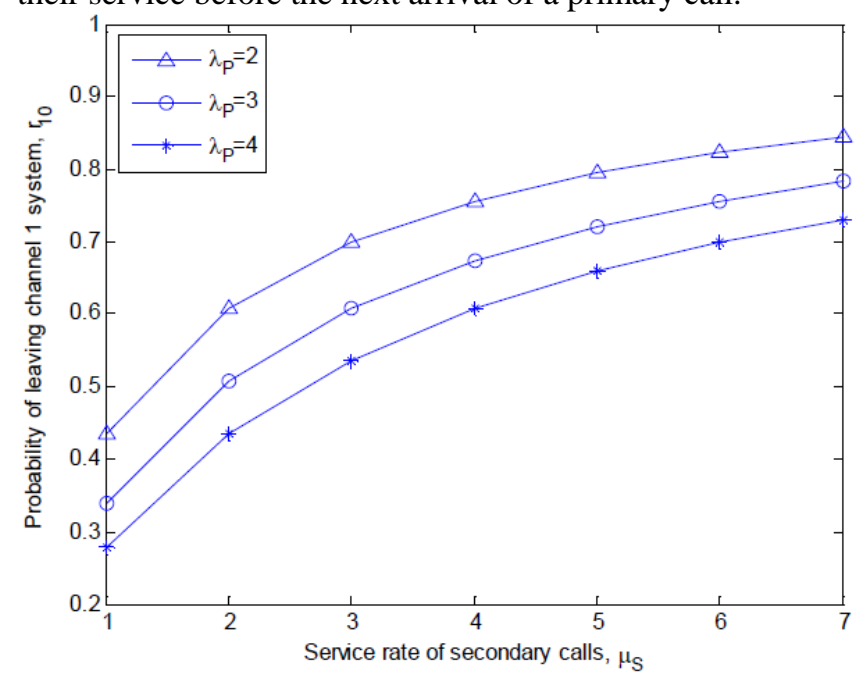

Figure 3. Prob. of leaving system vs. service rate $\mu_{S}$

In Fig. 4, we show how the mean number of secondary calls at channel 1 system, $L_{1}$, change with respect to the secondary call external arrival rate (via the test variable $\gamma_{S}$ ) for different values of $\lambda_{P}$ and $\mu_{S}$. We observe that $L_{1}$ increases as $\gamma_{S}$ or $\lambda_{P}$ increases and decreases as $\mu_{S}$ increases. As $\lambda_{P}$ is increased, the spectrum is used more frequently by primary calls, leading to fewer opportunities for secondary calls to use the channel. Conversely, an increase in $\mu_{S}$ leads to more opportunities for other secondary calls in the queue to use the channel.

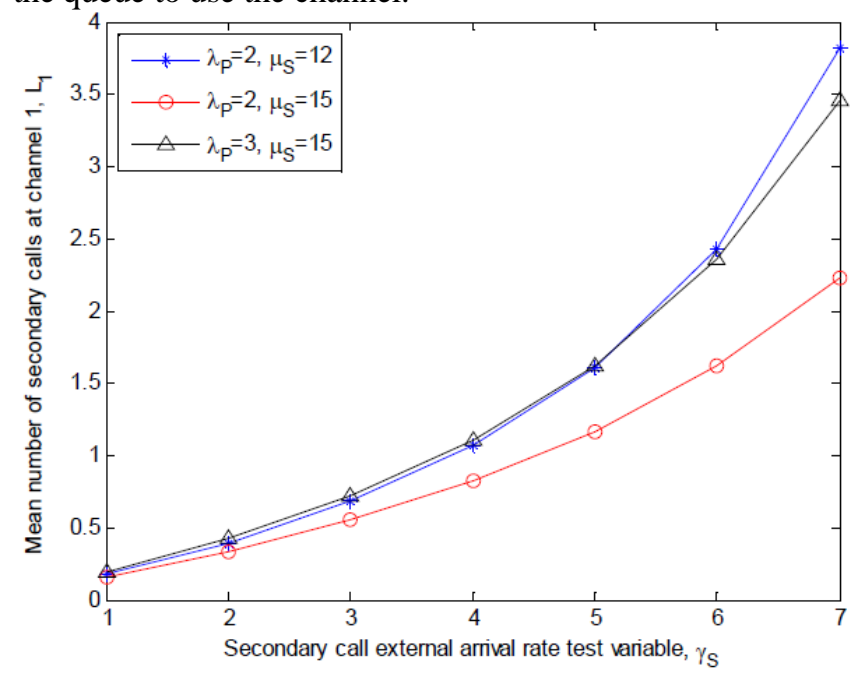

Figure 4. Mean number of secondary users in system
Fig. 5 shows the contribution factor of secondary calls at channel 1 system, $\eta_{1}$. We observe that $\eta_{1}$ increases as $\gamma_{S}$ increases and decreases as $\mu_{S}$ increases. As $\gamma_{S}$ is increased, the system utilization obviously increases and the contribution due to secondary calls increases. While the increase of $\mu_{s}$ leads to more chance of the completion of the secondary call and thus less time of utilizing the channel. We also observe $\eta_{1}$ changes little with respect to $\lambda_{P}$. This is because an increase of $\lambda_{P}$ on one hand reduces the chance of secondary call's contribution under low secondary traffic, on the other hand increases the chance of secondary call's contribution under high secondary traffic, which leads to more waiting calls in the queue and thus more chance to reconnect back to system.

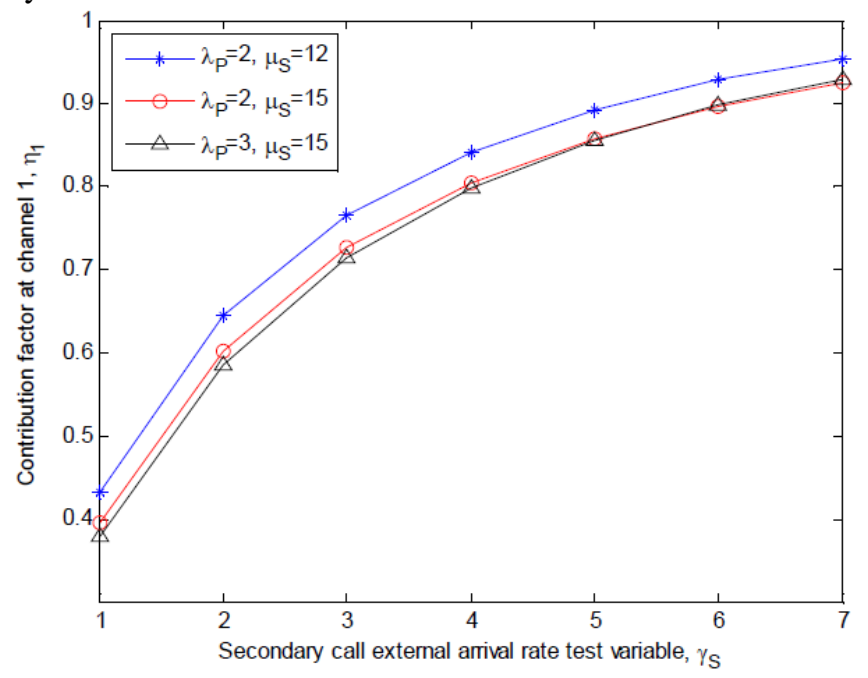

Figure 5. Contribution factor of secondary calls

\section{CONCLUSIONS}

We presented an analytic performance model of a delay-tolerable CR network, where secondary calls opportunistically share the channel resources with primary calls via spectrum sensing. When a secondary user detects the presence of a primary call, it will switch out to another channel with some routing probability. A queue is maintained in each channel for holding the secondary calls due to channel-busy. We derived explicit solutions for the system model in terms of generating functions and provided numerical validation. The analytical results derived in this paper can be used for different aspects of design and performance evaluation of cognitive radio data networks.

\section{ACKNOWLEDGEMENT}

This work was supported in part by the National Natural Science Foundation of China under Grant No. 61462020. 


\section{REFERENCES}

[1] S. Tang, B.L. Mark, Modeling and Analysis of Opportunistic Spectrum Sharing with Unreliable Spectrum Sensing, IEEE Transactions on Wireless Communications, 8 (2009) 1934-1943.

[2] S. Atapattu, C. Tellambura, H. Jiang, Energy Detection Based Cooperative Spectrum Sensing in Cognitive Radio Networks, IEEE Trans. Wireless Comm., 10 (2011) 1232-1241.

[3] H. Kim, K.G. Shin, Efficient discovery of spectrum opportunities with mac-layer sensing in cognitive radio networks, IEEE Trans. Mobile Comput., 7 (2008) 533-545.

[4] S. Tang, Reconnection Analysis for a Cognitive Radio Network with unreliable Sensing, Wireless Personal Communications, 69 (2013) 299-305.

[5] L.C. Wang, C.W. Wang, F. Adachi, Load-Balancing Spectrum Decision for Cognitive Radio Networks, IEEE J. Sel. Area. Comm., 29 (2011) 757-769.
[6] B. Wang, Y. Wu, K.J.R. Liu, Game theory for cognitive radio networks: An overview, Computer Networks, 54 (2010) 2537-2561.

[7] A. Tabassam, M.U. Suleman, Game theory in wireless and cognitive radio networks - coexistence perspective, IEEE Symposium on wireless tech. \& app., (2012) 177-181.

[8] M. Abdelbar, B. Tranter, T. Bose, Cooperative modulation classification of multiple signals in cognitive radio networks, 2014 IEEE Int'l Conf. on Comm. (ICC'14), pp. 1483-1488.

[9] G. Matsuia, T. Tachibana, Y. Nakamurac, K. Sugimotoa, Distributed power adjustment based on control theory for cognitive radio networks, Computer Networks, 57 (2013) 3344-3356.

[10] P.G. Harrison, N.M. Patel, Performance Modelling of Communication Networks and Computer Architectures. Boston, MA, 1993.

[11] U. Yechiali, P. Naor, Queuing problems with heterogeneous arrivals and service, Operations Research, 19 (1971) 722-734. 